\title{
Bayesian Model Averaging with Temporal Correlation for Time Series Forecasts
}

\author{
KOSUKE ONO ${ }^{\mathrm{a}, \mathrm{b}}$ \\ ${ }^{a}$ Meteorological Research Institute, Japan Meteorological Agency, Tsukuba, Japan \\ ${ }^{\mathrm{b}}$ Numerical Prediction Development Center, Japan Meteorological Agency, Tsukuba, Japan
}

(Manuscript received 30 January 2021, in final form 3 June 2021)

\begin{abstract}
This study extends Bayesian model averaging (BMA) to a form suitable for time series forecasts. BMA is applied to a three-member ensemble for temperature forecasts with a 1-h interval time series at specific stations. The results of such an application typically have problematic characteristics. BMA weights assigned to ensemble members fluctuate widely within a few hours because BMA optimizations are independent at each lead time, which is incompatible with the spatiotemporal continuity of meteorological phenomena. To ameliorate this issue, a degree of correlation among different lead times is introduced by the extension of latent variables to lead times adjacent to the target lead time for the calculation of BMA weights and variances. This extension approach stabilizes the BMA weights, improving the performance of deterministic and probabilistic forecasts. Also, an investigation of the effects of this extension technique on the shapes of forecasted probability density functions showed that the extension approach offers advantages in bimodal cases. This extension technique may show promise in other applications to improve the performance of forecasts by BMA.
\end{abstract}

KEYWORDS: Ensembles; Forecasting techniques; Probability forecasts/models/distribution

\section{Introduction}

In recent studies of meteorological predictability, the wellknown limitations of deterministic forecasts have led to efforts to improve probabilistic forecasting skills in weather forecasts (e.g., Palmer 2018). For that purpose, reliable ways to create an accurate probability density function (PDF) from an ensemble forecast are essential. In general, a PDF derived from a raw ensemble forecast is underdispersive, which means that it underestimates forecast errors, and is to be corrected. Therefore, the technique used to calibrate a PDF created from an ensemble forecast is key to more accurate weather prediction.

Bayesian model averaging (BMA) is a useful way to unite multiple statistical models. In the meteorological context, Raftery et al. (2005) first applied BMA to dynamical models instead of statistical models for surface temperature prediction. It has since found application in areas such as precipitation (e.g., Sloughter et al. 2007; Schmeits and Kok 2010; Ji et al. 2019) and wind forecasts (e.g., Sloughter et al. 2013; Eide et al. 2017) and has improved the skill of visibility (Chmielecki and Raftery 2011) and tropical cyclone intensity (Song et al. 2018) forecasts. Also, Bishop and Shanley (2008) studied BMA for extreme weather events. The objectives of BMA for ensemble predictions are to calibrate PDFs generated from raw ensembles, to create PDF-associated deterministic forecasts (e.g., forecasts corresponding to the weighted mean, median, and mode of a PDF), and probabilistic forecasts with a PDF. Therefore, BMA plays an important role in improving the accuracy of both deterministic and probabilistic predictions.

The Japan Meteorological Agency (JMA) has been investigating the blend of multiple forecast guidance to further improve deterministic forecasts and to create new probabilistic products

Corresponding author: Kosuke Ono, onok@mri-jma.go.jp
(Mori et al. 2021). In this study, BMA was applied to temperature time series forecasts with a 1-h interval in a three-member ensemble as one such trial. Whereas previous studies have applied BMA to a single-time forecast or a time series forecast with a long time interval, such as $24 \mathrm{~h}$ (Wilson et al. 2007; Schmeits and Kok 2010; Eide et al. 2017; Ji et al. 2019), no studies have applied BMA to a time series forecast with a short time interval.

One problem peculiar to time series forecasts with a short time interval is that the weights of ensemble members may fluctuate widely over a few hours because the BMA optimization (detailed in section 2) is performed independently for each lead time. The weights assigned to the contributions of individual ensemble members to a Gaussian mixture PDF reflect the performance of the individual models. Compared with the performance of each model, BMA weight fluctuations are large (see Fig. 5). Also, given the spatiotemporal continuity of meteorological phenomena, short-term fluctuations in BMA weights are unnatural. Such rapid weight fluctuations make it difficult for forecasters to understand and are not appropriate for operational use. Berrocal et al. (2007) pointed out the lack of spatial correlation due to the independent optimization of each location in BMA and introduced spatial correlations between different locations. However, no studies have considered temporal correlations between forecast lead times for BMA forecasts.

In connection with the lack of correlation by independent optimizations, approaches to the lack of intervariable correlation by postprocessing include copulas (e.g., Möller et al. 2013; Wilks 2014). In this study, we propose the following BMA-specific method to introduce temporal correlation.

Publisher's Note: This article was revised on 2 September 2021 to replace the original Fig. 4, which had legends that were incomplete when originally published, and to fix a typographical error in the third paragraph in section 5 . 


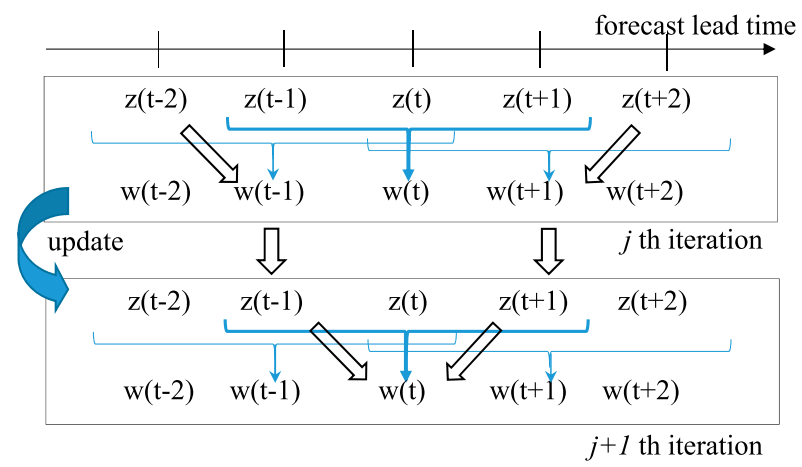

FIG. 1. Schematic showing information propagation from outside the extended window at the lead time $t$ of an experiment with $X=$ 1. Blue lines and arrows represent extension approach in BMA, and black arrows indicate information propagation. Effects of BMA variance are ignored.

To stabilize the large weight fluctuations, the use of latent variables (see in section 2) for the estimation of BMA weights and variances were extended to adjacent lead times before and after the forecast lead time. This approach aims at the introduction of correlation to independent BMA optimization at each lead time, also improved the deterministic and probabilistic performances. Moreover, the impacts of this approach on characteristics of bimodality in predicted PDF were explored, since the changes of weight characteristics are directly linked to PDF shapes.

The structure of this paper is as follows. Section 2 briefly introduces BMA with a Gaussian mixture model and formulates the extension technique. Section 3 describes the experimental setting. Section 4 compares the performances and predicted PDFs between the original and the modified BMA. Section 5 summarizes the study and discusses the possibilities of applying this new approach to BMA for other forecasting techniques.

\section{BMA and its extension}

\section{a. BMA with a Gaussian mixture model}

The calculated PDF $p(y)$ for meteorological element $y$ is described as follows:

$$
p(y)=\sum_{k=1}^{K} w_{k} g_{k}\left(f_{k}, \sigma\right),
$$

where $K$ is the number of ensemble members, $w_{k}$ is the weight assigned to the $k$ th member, $g_{k}$ is the $k$ th member's Gaussian distribution with mean $f_{k}$, which is substituted for the $k$ th ensemble member forecast, and $\sigma$ is the standard deviation of $g_{k}$. For simplicity, $\sigma$ is a common value for all members following previous studies (e.g., Wilson et al. 2007). From Eq. (1), the Gaussian mixture PDF $p(y)$ is a weighted mean of each Gaussian $g_{k}$. The parameters $w_{k}$ and $\sigma$ are calculated from a maximum likelihood estimate. Because it is difficult to optimize Eq. (1) directly, the expectation-maximization algorithm (Dempster et al. 1977;

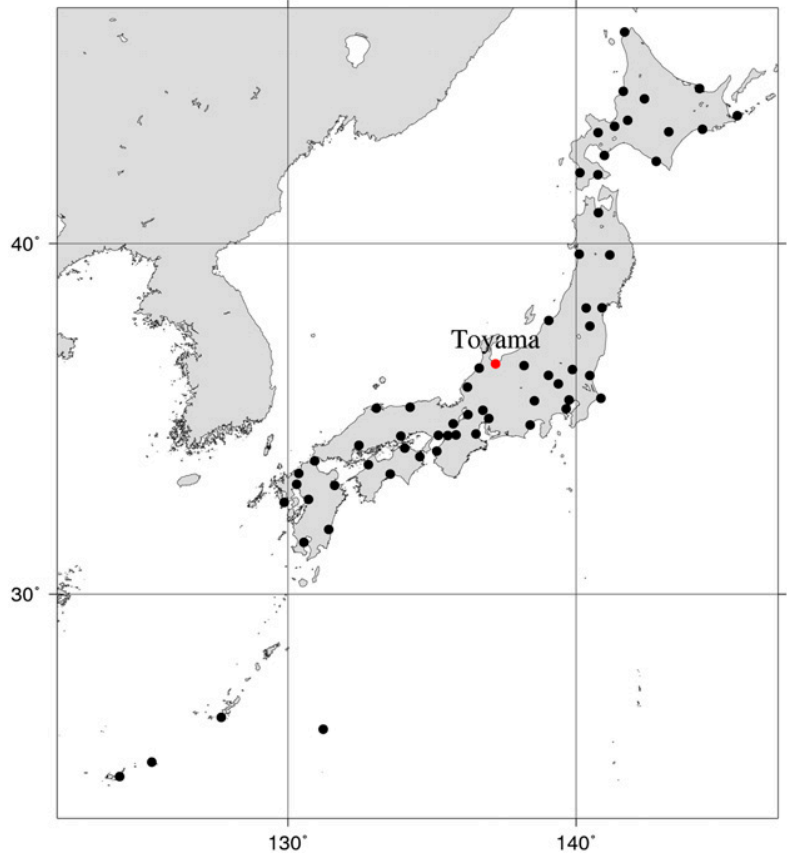

FIG. 2. Map of Japan showing meteorological observation stations used in this study. Toyama station (red circle) is used for a case study in section 4 .

Bishop 2010) is applied to the estimation of parameters in Eq. (1).

The parameter estimation for station $s$ at forecast lead time $t$ is now considered by the use of each member forecast $f_{k s t}$ initialized during the past $N$ days ( $N$ is called "training period," which is a hyperparameter of BMA) and corresponding observations $y_{s t}$. Furthermore, $f_{k s t}$ is assumed to have been corrected for bias; thus, it corresponds to a guidance forecast for the $k$ th member forecast.

The expectation-maximization algorithm is an iterative algorithm that introduces latent variable $z$, an unobservable variable that corresponds to $s, t$ of the $k$ th member in the past $n$ days. The $j$ th iteration is defined as

$$
z_{k s t n}^{(j)}=\frac{w_{k s t}^{(j-1)} g_{k s t n}\left(f_{k s t n} \mid \sigma_{s t}^{(j-1)}\right)}{\sum_{i=1}^{K} w_{i s t}^{(j-1)} g_{i s t n}\left(f_{i s t n} \mid \sigma_{s t}^{(j-1)}\right)},
$$

where $z_{k s t n}^{(j)}$ is calculated by only $f_{k s t n}, w_{k s t}^{(j-1)}$, and $\sigma_{s t}^{(j-1)}$. By using $z_{k s t n}^{(j)}$, the weight of the $k$ th member $w_{k s t}^{(j)}$ is derived by averaging $z_{k s t n}^{(j)}$ for the past $N$ days:

TABLE 1. Models used in this study.

\begin{tabular}{llcl}
\hline \hline Member & Source & Driving model & \multicolumn{1}{c}{ Resolution } \\
\hline GSM & JMA & $\begin{array}{c}\text { Global spectrum } \\
\text { model }\end{array}$ & TL959 $(\sim 20 \mathrm{~km})$ \\
MSM & JMA & $\begin{array}{c}\text { Mesoscale model } \\
\text { (regional model })\end{array}$ & $5 \mathrm{~km}$ \\
NCEP & NCEP & $\begin{array}{c}\text { Global model } \\
\text { T1534 }(\sim 13 \mathrm{~km})\end{array}$ \\
\hline
\end{tabular}



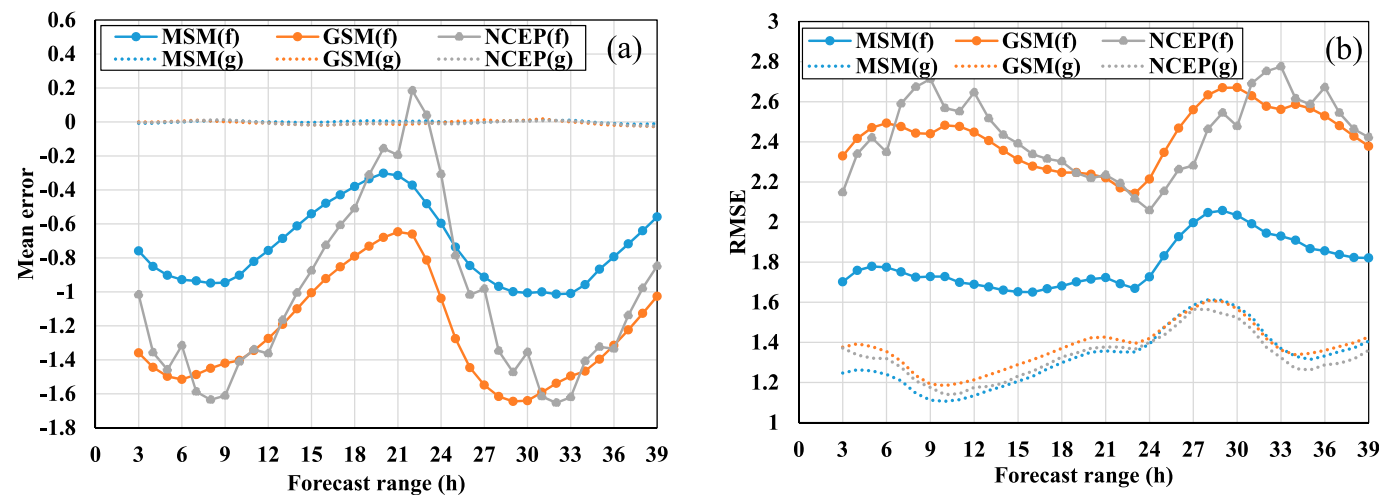

FIG. 3. (a) Mean errors and (b) RMSEs of raw forecasts (solid lines labeled "f"), and guidance forecasts (dotted lines labeled " $\mathrm{g}$ ") from the MSM, GSM, and NCEP model for forecasts of 3-39 h. Calculated from 63 stations and 360 initial values. Forecast range is at 1 -h intervals.

$$
w_{k s t}^{(j)}=\frac{1}{N} \sum_{n=1}^{N} z_{k s t}^{(j)} .
$$

Also, the standard deviation $\sigma_{s t}^{(j)}$, which is common to all members, is determined as follows:

$$
\left(\sigma_{s t}^{(j)}\right)^{2}=\frac{1}{N} \sum_{n=1}^{N}\left[\sum_{i=1}^{K} z_{i s t n}^{(j)}\left(y_{s t n}-f_{i s t n}\right)\right] .
$$

Equation (2) is based on Bayes's theorem, where the prior distribution $w_{k s t}^{(j-1)}$ is updated using the likelihood function $g_{k s t n}\left(f_{k s t n} \mid \sigma_{s t}^{(j-1)}\right)$. The posterior distribution of $w_{k s t}^{(j)}$ is updated via $z_{k s t n}^{(j)}$ in Eq. (3).

Using the weight $w_{k s t}^{(j)}$ and standard deviation $\sigma_{s t}^{(j)}$, the loglikelihood at $j$ th iteration $\ell_{s t}^{(j)}$ is

$$
\ell_{s t}^{(j)}=\sum_{n=1}^{N} \log \left[\sum_{k=i}^{K} w_{i s t}^{(j)} g_{i s t n}\left(f_{i s t} \mid \sigma_{s t}^{(j)}\right)\right]
$$

To estimate parameters for many stations at multiple forecast lead times for a time series forecast, Eq. (5) is summed over variables $s$ and $t$. The iteration is performed until the log-likelihood converges. Each optimization is independent at each station and lead time.

After the iteration reach convergence, the weights $w_{k s t}$ and standard deviation $\sigma_{k s t}$ are used to estimate the Gaussian mixture or calculate the weighted mean forecast as well as serving as the first iteration in the next BMA parameter estimation.

\section{b. Extension approach}

The BMA weights of ensemble members fluctuate widely for a time series forecast (see Fig. 5a) because the optimizations at each lead time are independent and uncorrelated. Therefore, to introduce a degree of correlation between lead times, the population of latent variables is extended to adjacent lead times before and after the target lead time.

The latent variable of $z$ is calculated as following Eq. (2). Then, on the estimation of $w_{k s t}^{(j)}$ and $\sigma_{s t}^{(j)}$, the use of data adjacent before and after $X$ hours is considered. For simplicity, the forecast time interval is assumed to be $1 \mathrm{~h}$. Introducing the suffix $x$ to indicate the forecast lead time before and after $x$ hours, Eqs. (3) and (4) become, respectively,
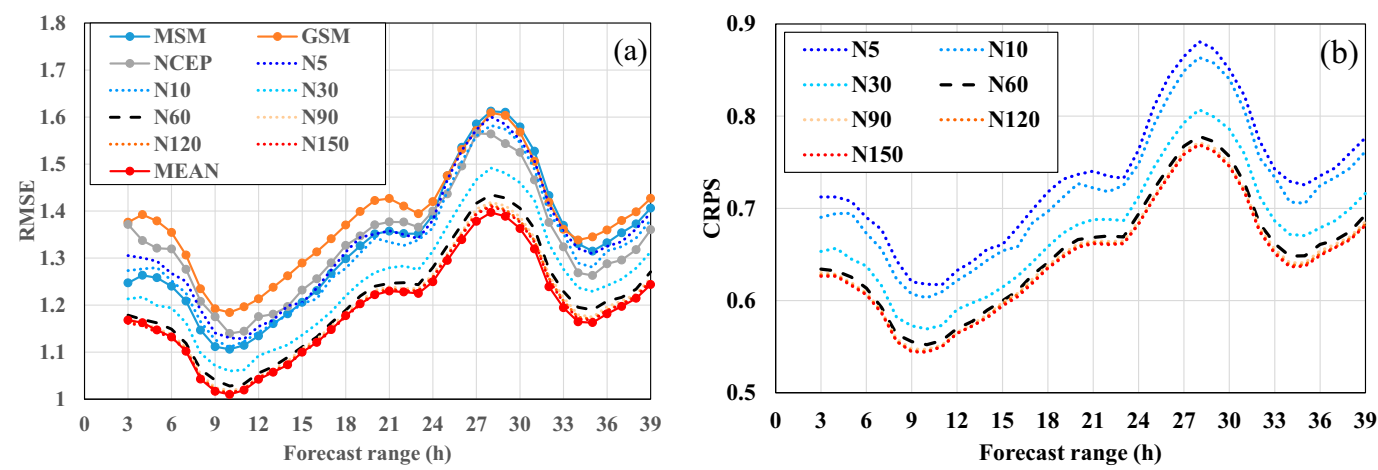

FIG. 4. (a) RMSE and (b) CRPS of each guidance forecast, ensemble mean forecast (MEAN), and weighted mean forecast by BMA with different values of $N$ for forecasts of 3-39 h. Calculated from 63 stations and 360 initial values. Forecast range is at 1 -h intervals. 

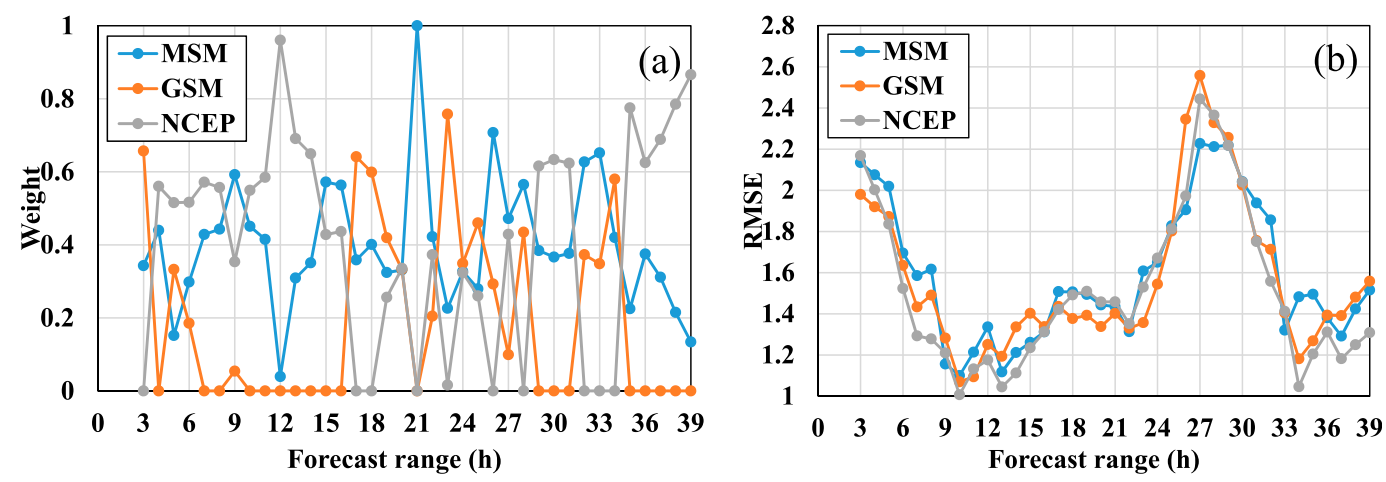

FIG. 5. (a) Weight coefficients and (b) RMSE in training period of ensemble members in experiment N60 for each forecast lead time from 3 to $39 \mathrm{~h}$ at Toyama station initialized at 0000 UTC 9 Sep 2018.

$$
w_{k s t}^{(j)}=\frac{1}{N(2 X+1)} \sum_{x=-X}^{X} \sum_{n=1}^{N} z_{k s(t+x) n}^{(j)}
$$

and

$\left(\sigma_{s t}^{(j)}\right)^{2}=\frac{1}{N(2 X+1)} \sum_{x=-X}^{X}\left\{\sum_{n=1}^{N}\left[\sum_{i=1}^{K} z_{i s(t+x) n}^{(j)}\left(y_{s(t+x) n}-f_{i s(t+x) n}\right)\right]\right\}$.

This extension does not increase the stored data, which is important for operational systems with limited computer resources. Also, the coding is easy, as it only extends the calculations of weights and variance in Eqs. (6) and (7).

The information at each lead time propagates beyond the range of $X$, as the iteration progresses shown in Fig. 1, which stabilizes the weight fluctuations seen in section 4 . Due to the limited number of samples available, $X$ is limited near the edges of the forecast range. For example, in the case of a target lead time of $38 \mathrm{~h}$ and the end of a forecast lead time of $39 \mathrm{~h}$ when $X=3$, our method can be extended to $3 \mathrm{~h}$ before the target time, but only $1 \mathrm{~h}$ after the target time, resulting in an asymmetric extension. The effect of this asymmetric extension on forecast performance is discussed in section 4.

In this extension, the hyper-parameter includes the extended forecast range $X$ in addition to $N$. This study also investigates the sensitivity of $X$ to BMA performance.

\section{Experimental design}

\section{a. BMA for a time series temperature prediction}

For forecasts of surface temperature, 63 Automated Meteorological Data Acquisition System (AMeDAS; JMA 2019) operated by JMA were targeted (Fig. 2).

Guidance forecasts were generated from three-model forecasts for each station: the Global Spectrum Model (GSM) and Mesoscale Model (MSM), computed by JMA, and the global model of the U.S. National Centers for Environmental Prediction (NCEP) (Table 1). Therefore, this study employed a three-member ensemble system.

The verification period was from 0000 UTC 1 December 2017 to 0000 UTC 30 November 2018, for a total of 360 initial times (excluding 5 days when the forecast data were not stored). The MSM forecast range was $39 \mathrm{~h}$ during this period, and the guidance forecasts of the GSM were generated $3 \mathrm{~h}$ after each initial time. Thus, BMA forecasts in the range from 3 to $39 \mathrm{~h}$ were produced at 1 -h intervals. All forecasts are initialized at 0000 UTC each day at each station. As the local standard time (LST) in Japan is $9 \mathrm{~h}$ ahead of UTC, 0000 UTC corresponds to 0900 LST.

This study investigated the sensitivity of ensemble member weights and forecast performance to the length of training period $N$. The training period $N$ was set to $5,10,30,60,90$, 120 , and 150 days in experiments labeled $\mathrm{N} 5, \mathrm{~N} 10, \ldots$, $\mathrm{N} 150$, respectively. Also, the extension technique was applied to the N60 experiments, and the value of $X$ was set to 1,2 , and $3 \mathrm{~h}$, which were labeled N60X1, N60X2, N60X3, respectively.

\section{b. Verification methods}

Deterministic and probabilistic forecasts were verified by using AMeDAS data at each station as reference values. To verify the deterministic forecasts obtained by BMA, the root mean squared error (RMSE) was calculated for weighted mean forecasts. The simple ensemble means of the three guidance forecasts were also compared. To verify the probabilistic forecasts, the experiments were compared by using

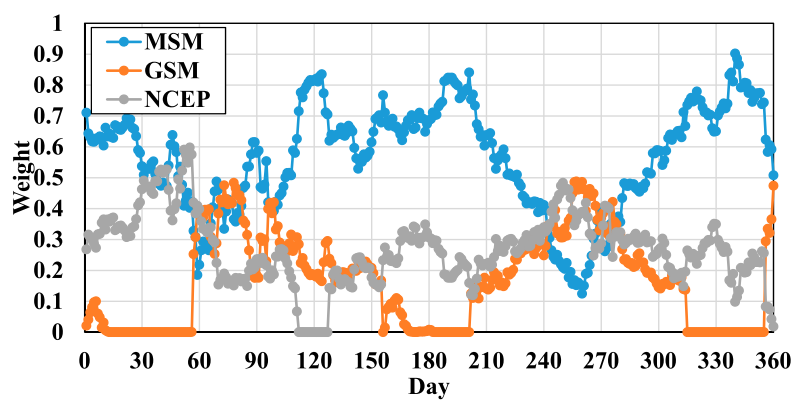

FIG. 6. Time series of weight coefficients of ensemble members in experiment N60 from each guidance forecast at Toyama station with a 24-h lead time. The period is from $1 \mathrm{Dec} 2017$ to 30 Nov 2018. 


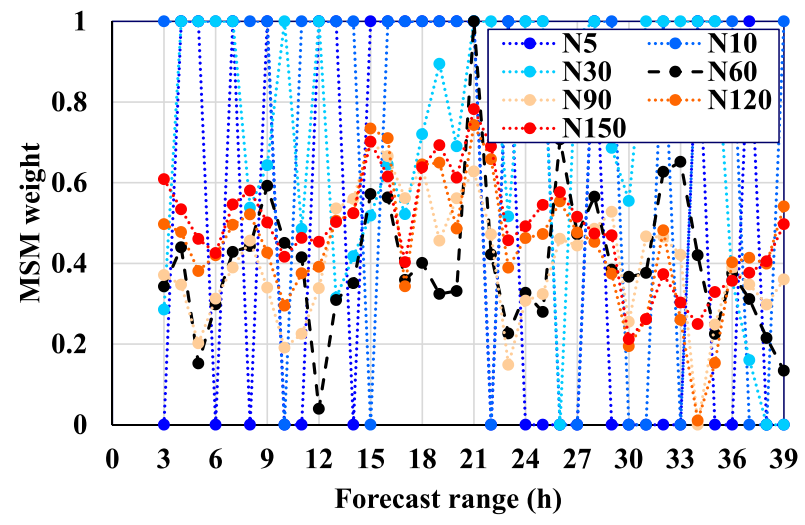

FIG. 7. MSM weight coefficients of forecast lead time from 3 to $39 \mathrm{~h}$ at Toyama station initialized at 0000 UTC 9 Sep 2018 for different values of $N$.

the continuous rank probability score (CRPS; e.g., Hersbach 2000), which measures the differences between forecasted and observed cumulative density functions (CDFs). For meteorological element $x$, the CDFs of forecasts and observations are designated as $p(x)$ and $o(x)$, respectively. By using the Heaviside step function

$$
H(x) \equiv\left\{\begin{array}{ll}
0 & (\text { for } \quad x<0) \\
1 & (\text { for } \quad x \geq 0)
\end{array},\right.
$$

$o(x)$ is defined using observation $a$ :

$$
o(x-a) \equiv\left\{\begin{array}{ll}
0 & (\text { for } \quad x<a) \\
1 & (\text { for } \quad x \geq a)
\end{array} .\right.
$$

Using the Gaussian mixture PDF $p(y)$ predicted from Eq. (1), the CRPS is defined as

$$
\mathrm{CRPS} \equiv \frac{1}{N} \sum_{i=1}^{N}\left[p_{i}(y)-o_{i}(x)\right]^{2} d x
$$

The CRPS takes values greater than or equal to zero, with zero signifying a perfect forecast.

\section{c. Bias corrections in raw forecasts}

Raw forecast values that are linearly interpolated to individual stations have a forecast bias for several reasons, such as differences between modeled and the actual orography. Therefore, a Kalman filter was used to remove bias at each station, each lead time, and each model. Kalman filters have been operationally adopted by the JMA (JMA 2019) for the MSM and GSM, and a Kalman filter was used to correct the bias of NCEP global model forecasts in the same way as for the GSM. Figure 3 shows the effect of Kalman filtering on mean errors and RMSEs between the raw model forecasts and the guidance forecasts. Although each raw forecast had a negative bias at almost all forecast ranges, those biases were removed for the guidance forecasts. Also, the RMSEs of the guidance forecasts were greatly reduced compared to those of the raw forecasts.

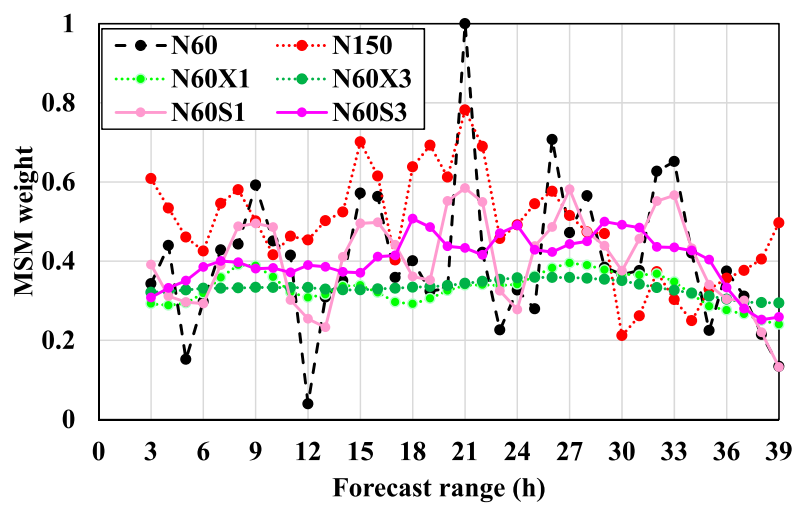

FIG. 8. MSM weight coefficients for forecast lead times of 3-39 h at Toyama station initialized at 0000 UTC 9 Sep 2018 from experiments N60X1, N60X3, N60S1, and N60S3 in addition to N60, N120, and N150.

\section{Results}

\section{a. Performance of the original BMAs for time series forecasts}

Previous studies have documented the relationship between the training period length $N$ and forecast performance with a specific lead time (e.g., Raftery et al. 2005; Wilson et al. 2007; Sloughter et al. 2007, and so on). In this study, such sensitivity was investigated for a time series forecast with a 1-h interval. Also, the sensitivity of $X$ to BMA weights was researched.

The BMA weighted mean forecasts, excluding N5 and N10, were improved compared with the individual guidance forecasts in terms of RMSEs, and also improved with increasing $N$ up to $N=60$ in terms of RMSEs and CRPSs (Fig. 4). These improvements with increasing $N$ are consistent with the findings of previous studies using temperature forecasts (see Fig. 7 of Raftery et al. 2005). However, no clear improvements were seen in experiments in which $N$ exceeded 60 .

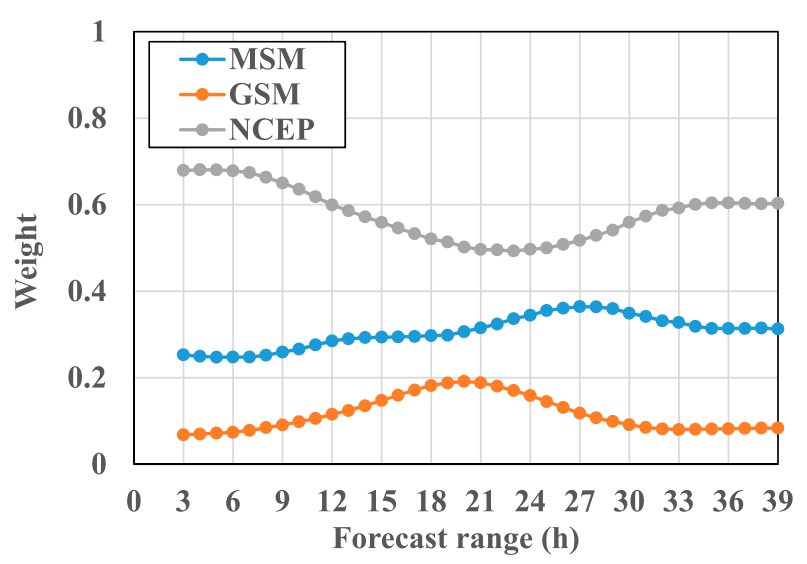

FIG. 9. Weight coefficients of ensemble members in experiment $\mathrm{N} 60 \mathrm{X} 3$ for each forecast lead time from 3 to $39 \mathrm{~h}$ at Toyama station initialized at 0000 UTC 9 Sep 2018. 


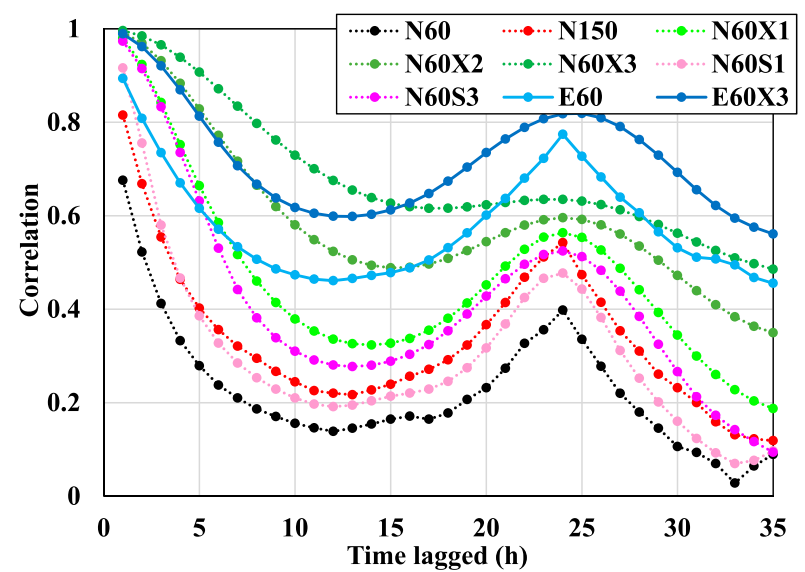

FIG. 10. Time-lagged correlation coefficients of MSM weight and forecast error (E60, E60X3, see text) calculated from all stations and all initial times in each experiment.

The ensemble mean forecast had the smallest RMSE among all of the forecasts (Fig. 4a). One possible reason for the relatively less performance of the BMA forecasts appears in Fig. 5, which shows the weight coefficients and RMSEs in the training period at Toyama station (Fig. 2) from three models initialized on 9 September 2018. At most lead times, the weight of one ensemble member was 0.0. Thus, the ensemble size in BMA was effectively two rather than three. The relationship between this weight characteristic and the less performance of the BMA weighted mean is discussed in section $4 \mathrm{c}$, based on the results of the extended experiment.

The member weights also fluctuated widely as the lead time changed (Fig. 5a). For example, the GSM weights at lead times of 22,23 , and $24 \mathrm{~h}$ were $0.2,0.75$, and 0.35 , respectively. Such abrupt changes are incompatible with the spatiotemporal continuity of meteorological phenomena. Also, the GSM weights were 0.0 at lead times of $10-16$ and $30 \mathrm{~h}$, while the differences in RMSE (Fig. 5b) between GSM and others were about $0.1-0.2$ at lead times $10-16$ and almost 0.0 at lead time $30 \mathrm{~h}$. This result indicates that the relative magnitude relationship between weights and RMSEs is not consistent over lead times. This is because parameters are estimated independently at each lead time.

Figure 6 shows the daily BMA weights for Toyama station over the study period estimated for a lead time of $24 \mathrm{~h}$. As previous studies have shown (e.g., Fig. 2 of Wilson et al. 2007), a zero weight member with a specific period was seen in this study, too.

As for the intense weight fluctuations in a few hours shown in Fig. 5a, the sensitivity of the length of the training period $N$ to BMA weights was investigated. Figure 7 shows the weights assigned to the MSM with different $N$ initialized on 9 September 2018 at Toyama. The large weight fluctuations with time from zero to one were seen in N5 and N10, but such fluctuations were inclined to suppress in more than $N=30$. Also, in the experiment of $\mathrm{N} 150$, the weights ranged from 0.2 to 0.8 , suggesting that a long training period can improve the usefulness of BMA by suppressing intense weight fluctuations.

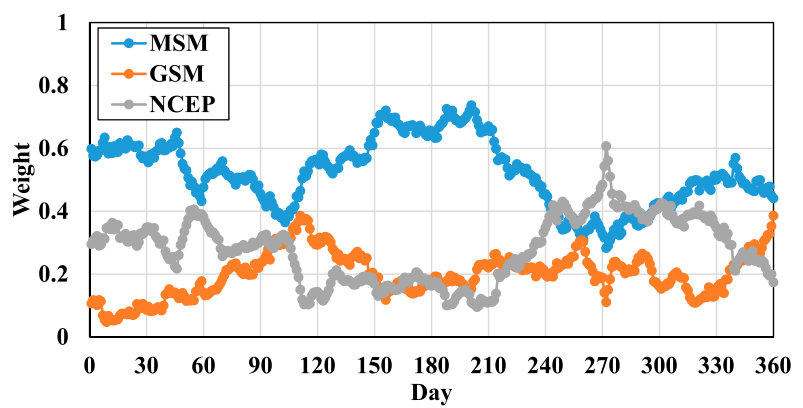

FIG. 11. Time series of weight coefficients of ensemble members in experiment N60X3 from each guidance forecast at Toyama station with a 24-h lead time. The period is from 1 Dec 2017 to 30 Nov 2018.

From these results, for aiming at 1) the introduction to a correlation between different lead times and 2) the increase in the training samples to suppress intense weight fluctuations, the use of latent variables to calculate weights and standard deviations is extended as Eqs. (6) and (7).

\section{b. Impact of extension technique on BMA weights}

This section shows the impact of the extension technique on the BMA weights. Another approach to subdue the wide weight fluctuations in BMA may be to employ a simple moving average of each weight among lead times. The experiments in which the moving average was based on one, two, and three adjoining hours (N60S1, N60S2, and N60S3) are also shown for comparisons.

Figure 8 shows the sensitivity of the length $X$ to the MSM weights in addition to moving average experiments. The MSM weights from N60 and N150 are also reposted in Fig. 8 for a comparison. Only 1-h extension (N60X1) made weights smoother compared with that of N150. Focusing on the difference in $X$, the weight was smoother from N60X3, but the impact was not significant. The weights from the N60S3

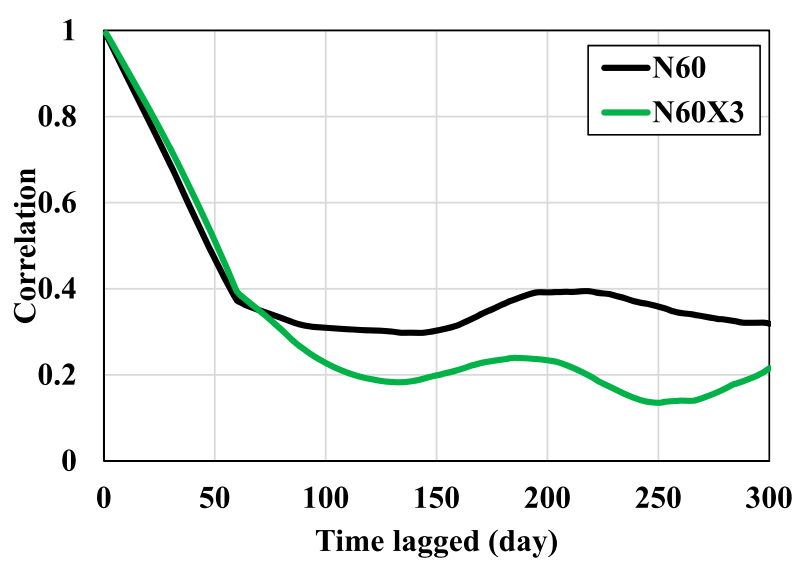

FIG. 12. Daily time-lagged correlation coefficients of MSM weight at a lead time of $24 \mathrm{~h}$ from experiment N60 and N60X3 calculated from all stations and all initial times. 

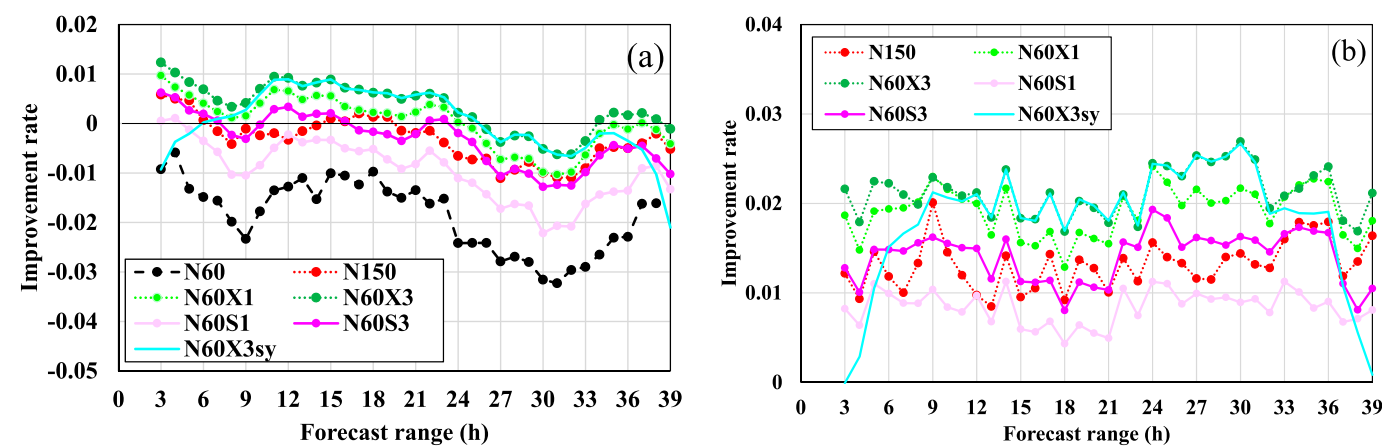

FIG. 13. Relative improvements for forecast lead times of 3-39h of (a) RMSE based on the ensemble mean forecast for experiments N60, N150, N60X1, N60X3, N60S1, N60S3, and N60X3sy and (b) CRPS based on experiment N60. See the text for details. Calculated from 63 stations and 360 initial values.

experiment also smoothed the distribution of the original weights, but minor fluctuations remained.

Figure 9 shows the effect of the extension technique on weight coefficients in the N60X3 experiments same as Fig. 5a. The introduction of correlation among adjacent weights greatly smoothed the weight fluctuations. Also, discarded members with a weight of 0.0 were not seen.

To clarify the impact on the correlation structure by the extension technique, the time-lagged correlation coefficients of the MSM weight in each experiment are shown in Fig. 10. The time-lagged correlation coefficients of the RMSE of MSM calculated in the training period are also shown in Fig. 10. E60 and E60X3 correspond the RMSE correlations calculated in the training period $N=60$ and $N=60$ with the extended window $X=3$, respectively.

The second peaks around 24 lagged-hour in Fig. 10 were shown from all experiments, reflecting the daily changes in surface temperature. The correlations of the N60 weight decreased rapidly with lag time. On the other hand, the correlation of the N150 increased, which was consistent with suppressing fluctuations in the weight coefficients by increasing $N$ in Fig. 7. Also, the correlation of N60 weights was smaller than that of E60 in all lead times, reflecting the intense weight fluctuations of the weights in Fig. 5a. The high correlations were found in N60X1, N60X2, and N60X3. The correlation of N60X3 is higher than that of E60X3 up to 15 lagged hours because of the information propagated from the outside of the extended window shown in Fig. 1. Compared with the moving average, the effect of the extension technique on correlation was strong.

Figure 11 shows the daily changes in individual weights in the N60X3 experiment, using the same record as in Fig. 6. The use of extension reduced the weight differences between members, and the daily variation in the range of weight was smaller. Besides, no members were assigned a weight of almost zero. Figure 12 shows the daily time-lagged correlation coefficients of the MSM weight at a lead time of $24 \mathrm{~h}$ from the N60 and N60X3 experiments calculated from all stations and all initials. Both correlations decreased linearly up to 60 lagged-day, which corresponds to the training period. The correlation in N60X3 is slightly higher than that of N60, indicating that the extension approach makes daily weights changes more gradual.

The impact of these BMA weight characteristics on forecast performances is shown in section $4 \mathrm{c}$.

\section{c. Impact of extension technique on BMA performances}

Figure 13 shows the relative improvement [e.g., Eq. (4) of Kunii et al. 2011], defined as the reduction of RMSE normalized by the RMSE of reference forecast,

$$
\text { relative improvement }=\frac{\mathrm{RMSE}_{\mathrm{ref}}-\mathrm{RMSE}_{\mathrm{tgt}}}{\mathrm{RMSE}_{\mathrm{ref}}},
$$

where $\mathrm{RMSE}_{\text {ref }}$ is the RMSE of a reference forecast and $\mathrm{RMSE}_{\mathrm{tgt}}$ is the RMSE of a targeted experiment. The relative improvement is also called "skill score" (Wilks 2011). A positive relative improvement indicates a performance superior to the reference forecast. The relative improvement can be defined for CRPS as well. The relative improvements of RMSE with respect to the ensemble mean forecast (Fig. 13a) were almost negative for the N60 and N150 experiments. The N60X1, N60X3, and N60S3 experiments yielded greater improvements than the N60 experiment. The time-averaged RMSEs are listed in Table 2, added the N60X2 and N60S2 experiments. The results showed that only the performances of the N60X2 and N60X3 experiments were superior to that of the ensemble mean forecast. The time-averaged RMSEs of experiments N60S1, N60S2, and N60S3 (Table 2) were larger than those of the ensemble mean forecast and experiment

TABLE 2. RMSEs averaged over the forecast range and over all initial times and stations. "MEAN" denotes ensemble mean forecast.

\begin{tabular}{ccccccccc}
\hline \hline MEAN & N60 & N150 & N60X1 & N60X2 & N60X3 & N60S1 & N60S2 & N60S3 \\
\hline 1.19226 & 1.21552 & 1.19669 & 1.19260 & 1.19008 & 1.18892 & 1.20483 & 1.19965 & 1.19674 \\
\hline
\end{tabular}


TABLE 3. RMSEs of N60 and N60X3 calculated by cases where the weight of one member in N60 is 0 or not. Calculated from all stations and all initial times.

\begin{tabular}{|c|c|c|c|c|c|c|}
\hline \multirow[t]{2}{*}{ Case } & \multicolumn{3}{|c|}{ N60 with weight $=0$} & \multicolumn{3}{|c|}{ N60 with weight $\neq 0$} \\
\hline & N60 & N60X3 & Difference & N60 & N60X3 & $\overline{\text { Difference }}$ \\
\hline RMSE & 1.20389 & 1.17743 & 0.02646 & 1.23168 & 1.20485 & 0.02683 \\
\hline
\end{tabular}

N60X1, although they were smaller than that of experiment N60. Therefore, the use of a moving average reduced errors compared with the N60 experiment but did not confirm an advantage over the simple ensemble mean forecast.

The relative improvements of CRPS with respect to N60 (Fig. 13b) show that the performances of all experiments were also superior to that of the N60 experiment. The improvement in CRPS implies that the changes in weight characteristics offered by the extension approach should influence the predicted PDF shapes, as discussed in section $4 \mathrm{~d}$.

As described in section 2, the latent variables available for this extension are limited near the edge of the forecast range, so the extension is asymmetric in the time direction. On the other hand, in the case of symmetrical extension, $X$ is smaller near the edge of the forecast range than in the asymmetric extension (e.g., for N60X3, the extension is limited to $X=1$ in a target lead time of $38 \mathrm{~h}$ ). Here, an $X$ symmetry experiment (N60X3sy) for N60X3 was performed to confirm the effect of asymmetric extension near the edge of the forecast range. Figure 13 also shows the relative improvements of RMSE and CRPS for N60X3sy, which are lower than those for N60X3, mainly at the edge of the forecast. This is because the available latent variables in N60X3sy are more limited closer to the edge of the forecast range than in N60X3.

As shown in Figs. 5a and 6, a zero weight for one member in the N60 experiment meant that the real ensemble size was reduced from 3 to 2 , and such cases accounted for $57 \%$ of the total. On the other hand, such zero weight cases were decreased significantly in N60X3 (Figs. 9 and 11), accounting for $1 \%$ of all cases. Therefore, a notable difference between the two experiments is whether there is a member with zero weights, and it would be interesting to see how much this difference contributed to the RMSEs. To investigate this, all cases were divided into two groups: those where the weight of one member was zero in N60, and those where it was not, and the RMSEs of N60 and N60X3 for each case were calculated (Table 3). The differences in RMSEs between the two experiments in both cases were about the same, about 0.026 , indicating that N60X3 had statistically smaller RMSEs, regardless of whether the weight of N60 was 0 or not.

\section{d. Changes to PDFs by extension technique}

This section focuses on the impacts of the extension approach on PDFs. The basic parameter that characterizes the width of a PDF is variance, and the most significant characteristic of a Gaussian mixture is its representation of the bimodality of a PDF. This section addresses the effects of extension on both variance and bimodality.
BMA variances (Raftery et al. 2005) is defined by

$$
\operatorname{Var}_{\text {BMA }}=\sum_{k=1}^{K} w_{k}\left(f_{k}-\sum_{i=1}^{K} w_{k} f_{k}\right)^{2}+\sigma^{2} .
$$

The first term on the right-hand side is the variance among members, and the second term is the variance within individual models, which is assumed to be the same for all three models in this study (see section 2). Figure 14 shows the sensitivity of BMA variances to $N$ and $X$, along with the sample variance calculated from the three guidance forecasts, which is defined by

$$
\operatorname{Var}=\frac{1}{k} \sum_{k=1}^{K}\left(f_{k}-\bar{f}\right)^{2},
$$

where $\bar{f}$ is the ensemble mean forecast from the three guidance forecasts.

The variance is large for N5 and N10, which take extreme weights of 0 or 1 , but becomes smaller as $N$ increases, and remains almost the same for N60 and N150.The effects of the extension technique, seen in experiment N60X3, was to make the BMA variance slightly larger and smoother in time.

The (calibrated) ensemble spread, which is defined by the squared root of Eq. (13) [Eq. (12)], and the RMSE of ensemble mean forecast are shown in Table 4. All values were averaged over the forecast range. The ideal ensemble has similar amplitudes between ensemble spread and RMSE of the ensemble mean from the spread-skill point of view. Spread deficiency compared with the RMSE of the ensemble mean forecast from

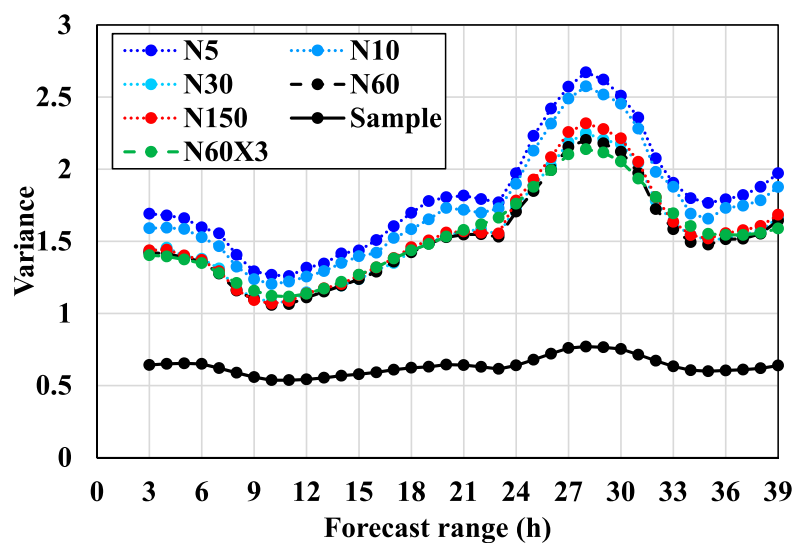

FIG. 14. BMA variance (see the text for details) for each experiment and the sample variance from three guidance forecasts ("Sample") for forecasts times of 3-39 h. Calculated from 63 stations and 360 initial values. 
TABLE 4. RMSE of ensemble mean, ensemble spread (SPD), and BMA-spreads from each experiment. Calculated from all stations and all initials.

\begin{tabular}{|c|c|c|c|c|c|c|c|c|c|c|c|}
\hline RMSE & SPD & N5 & N10 & N30 & N60 & N90 & N120 & N150 & N60X1 & N60X2 & N60X3 \\
\hline 1.19226 & 0.63687 & 1.34618 & 1.31621 & 1.24354 & 1.23377 & 1.24113 & 1.24567 & 1.25068 & 1.23504 & 1.23958 & 1.24208 \\
\hline
\end{tabular}

three guidance forecasts was dominant, consistent with the small variance seen in Fig. 14. On the other hand, such spread deficiency was ameliorated by the calibrated ensemble spread from the BMAs, especially in the experiment with larger $N$, which indicates that the calibration of the ensemble spread by BMA works well. In addition, the calibrated ensemble spreads from the BMA were slightly larger than the RMSE of ensemble forecast in the large $N$ and extended experiments. In experiments with small $N$, the variances are larger than the RMSE.

An important advantage of a Gaussian mixture PDF is that it expresses the bimodality of the predicted PDF. Figure 15 shows the frequency of bimodal PDFs occurrence as a percentage in addition to the RMSEs of the ensemble mean and BMA weighted mean forecasts calculated when the predicted PDF was bimodal. The percentage of bimodal PDFs is shown in relation to the total number of cases (the product of 37 lead times, 360 initial times, and 63 stations, minus those cases with missing observational data, for a total of 839034 cases). This figure shows that as $N$ increased, the number of bimodal PDF occurrences increased, and the RMSEs of all forecasts increased. The increase in the number of occurrences of bimodal PDFs saturated at N60, with a similar frequency of occurrence in the extended experiment.

The reason why the less frequency of bimodal PDFs small $N$ experiments is that it is difficult for PDFs to be bimodal due to the weights taking extreme values. This tendency is suppressed when $N$ increases, so the frequency of bimodal PDFs increases.

The RMSEs are small when $N$ or $X$ is small for the following reason. The accuracy of the ensemble mean does not depend on the shape of Gaussian mixture PDFs. Therefore, the increase in the RMSE of the ensemble mean when $N$ or $X$ is large indicates that the bimodal cases when $N$ or $X$ is large are less predictable (supported by the large RMSE of ensemble mean) than when $N$ or $X$ is small. This result means that predicted PDFs with small $N$ or $X$ can be bimodal, even if cases are predictable, caused by the spurious bimodal PDF.

An example of spurious PDF is seen in the case initialized at 0000 UTC 9 September 2018 at Toyama station (the same example as in Figs. 5 and 9). Figure 16 shows the Gaussian mixtures forecasted by the N60 and N60X3 experiments at a lead time of $9 \mathrm{~h}$. In the N60 experiment, the GSM weight was 0.05 (Fig. 5a), even though it yielded a result closer to the observation than MSM did. NCEP and MSM yielded temperature forecasts of about $19.5^{\circ}$ and $22.2^{\circ} \mathrm{C}$, respectively. This difference made the Gaussian mixture PDF bimodal (Fig. 16a). Because of this bimodality, the error of the BMA weighted mean forecast was larger than that of the ensemble mean forecast. On the other hand, in the N60X3 experiment, the weight of GSM was about 0.1 (Fig. 9). As the GSM weight, which was discarded in the N60 experiment, was ameliorated in the N60X3 experiment, the predicted PDF of the Gaussian mixture was unimodal, and the error of the weighted mean forecast was smaller than that of the N60 experiment. In addition, the error of the mode forecast was notably improved in the N60X3 experiment such that its temperature forecast (about $19.8^{\circ} \mathrm{C}$ ) was closer to the observation $\left(19.3^{\circ} \mathrm{C}\right)$ than the $\mathrm{N} 60$ forecast (about $22.0^{\circ} \mathrm{C}$ ).

The advantage of the extended approach was also confirmed by the comparison of RMSEs in Fig. 15. The RMSE of the weighted mean forecast (black line) was smaller with the extension approach (from N60X1 to N60X3) than in the original experiment (N60), a result of the extension approach's superiority in the bimodal cases.

\section{Summary and discussion}

In this study, BMA was applied to time series temperature forecasts with a 1-h interval of a three-member ensemble system to produce PDFs and improve deterministic forecasts. Since the optimizations in BMA were performed independently for each forecast lead time, the BMA weight coefficients fluctuated greatly in forecast ranges. Also, the relationship between weights and model performances was not consistent in different lead times. These results are specific for time series forecasts with a short time interval and caused less accuracy of the deterministic and probabilistic forecasts.

To reduce the wide fluctuations in BMA weights, the use of latent variables in BMA weight and variance calculations was

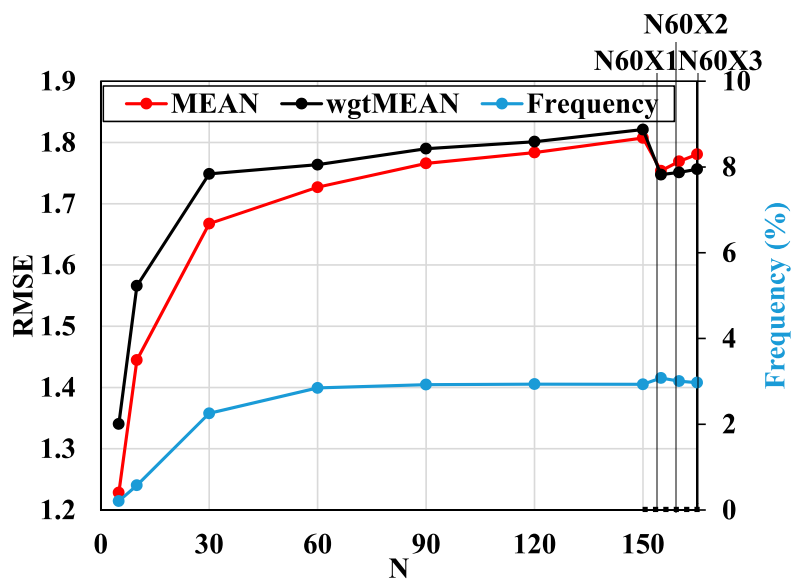

FIG. 15. RMSE of the ensemble mean forecast (MEAN) and BMA weighted mean forecast (wgtMEAN) derived from Gaussian mixtures as a function of $\mathrm{N}$. Also shown are RMSEs from N60X1, N60X2, and N60X3. Calculated from 63 stations limited to bimodal cases. The percentage of bimodal cases (Frequency; blue circles and line) is also shown. 

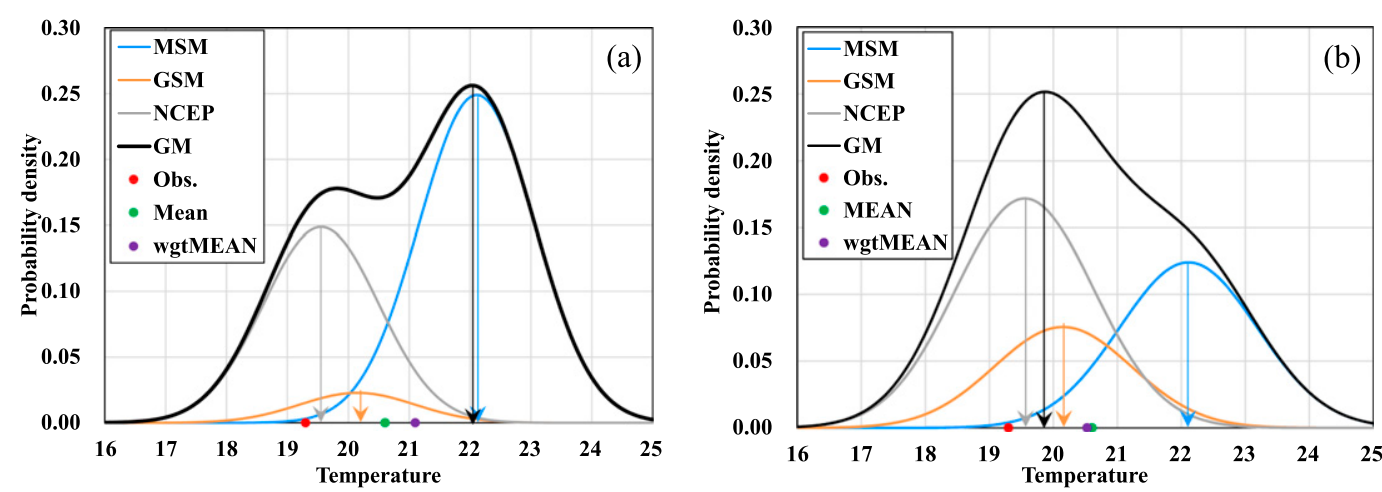

FIG. 16. Predicted Gaussian mixture PDF (GM; black) derived from experiment (a) N60 and (b) N60X3 with each ensemble member Gaussian at Toyama station with a lead time of $9 \mathrm{~h}$ initialized at 0000 UTC 9 Sep 2018. The observation (Obs.; red), the forecast from the ensemble mean (Mean; green), and the forecast from the BMA weighted mean (wgtMEAN; purple) are shown by circles. Arrows represent forecasted temperatures by each model and mode from GM.

extended to adjacent forecast lead times. This extension approach, which is an easy-to-implement method, introduced correlations of the BMA weights between each forecast lead time. The extension technique produced a much smoother distribution of BMA weights across the forecast range and improved performance of deterministic and probabilistic forecasts. Moreover, this extension reduced the number of bimodal PDFs in predictable cases.

For applications of the extension technique in more general cases, the time interval of forecasts may be longer than that used in this study. The forecast interval in many previous studies was $24 \mathrm{~h}$, as mentioned in section 1 . Thus, the question arises whether the extension approach improves accuracy when the data referenced as $X$ are not adjacent to the target lead time. An additional variant of experiment N60X1 was conducted that limited target lead time of $24 \mathrm{~h}$ and extended latent variables derived from 12- and 36-h lead time, apart from $12 \mathrm{~h}$. This experiment (TEST in Table 5) improved upon the N60 experiment in terms of RMSE. The result suggests that this extension technique is applicable to large interval forecasts, but further research is needed.

In this study, the results for the extended window up to $X=3$ were shown, but the effect of longer $X$ has not been investigated. As a trial, the experiment with $X=37$ (N60X37), i.e., with constant weights for each model in all forecast ranges, was conducted. The RMSE of N60X37 averaged over the forecast range and over all initial times and stations was 1.18913 which smaller than that of the ensemble mean (1.192 26 in Table 2). This result suggests a moderately weighted mean has better accuracy than a simple ensemble mean.

The extension approach was applied to time directions in this study; however, the extension may also be applied to spatial directions for the introduction of spatial correlations between different locations (Berrocal et al. 2007; Kleiber et al. 2011). For example, data from nearby stations or neighboring grid cells may be used in a BMA parameter estimation for a target station.
In this study, the temperature whose PDF follows a normal distribution is the target of the extended method, but we believe that the method can be applied to precipitation that follows a gamma distribution. This is because the algorithm for estimating the weights and variances of the BMA using latent variables in the gamma distribution (Sloughter et al. 2013; Liu and Xie 2014) is the same as for the normal distribution. However, the time scale of error correlation in precipitation forecast can be shorter than that of temperature, especially in heavy rain cases. Therefore, care should be taken in the extended range of $X$. In addition, since the meteorological disturbances that cause heavy rain are seasonally dependent (e.g., typhoons in summer), it may be interesting to extend the training period to the same season of other years (as described in Sloughter et al. 2007) and apply the extension described in this paper.

In addition, although the extended approach was applied to a small ensemble of three members in this paper, it would be interesting to confirm the effectiveness of the method on a larger ensemble. Thus, we conducted an additional experiment to increase the number of members to 33 by adding 10 perturbed members for three models with the Gaussian noise with a mean of 0 and standard deviation of 0.1 . The 33-member N60 experiment is denoted as N60-33, and the N60X3 experiment as N60X3-33.

Figure 17 shows the time series of the weights of each member from the 33-member experiments for the same case as in Fig. 5. In N60-33, the members with the highest weights vary from hour to hour. However, such members in N60X3-33 are consistent across the forecast range and aggregate into four specific members when focusing on weights above 0.1 .

TABLE 5. RMSEs averaged over all initial times and stations at the lead time of $24 \mathrm{~h}$. The TEST experiment incorporated extensions to the lead times of 12 and $36 \mathrm{~h}$.

\begin{tabular}{lccc}
\hline \hline MEAN & N60 & N60X1 & TEST \\
\hline 1.24974 & 1.27994 & 1.24945 & 1.26934 \\
\hline
\end{tabular}



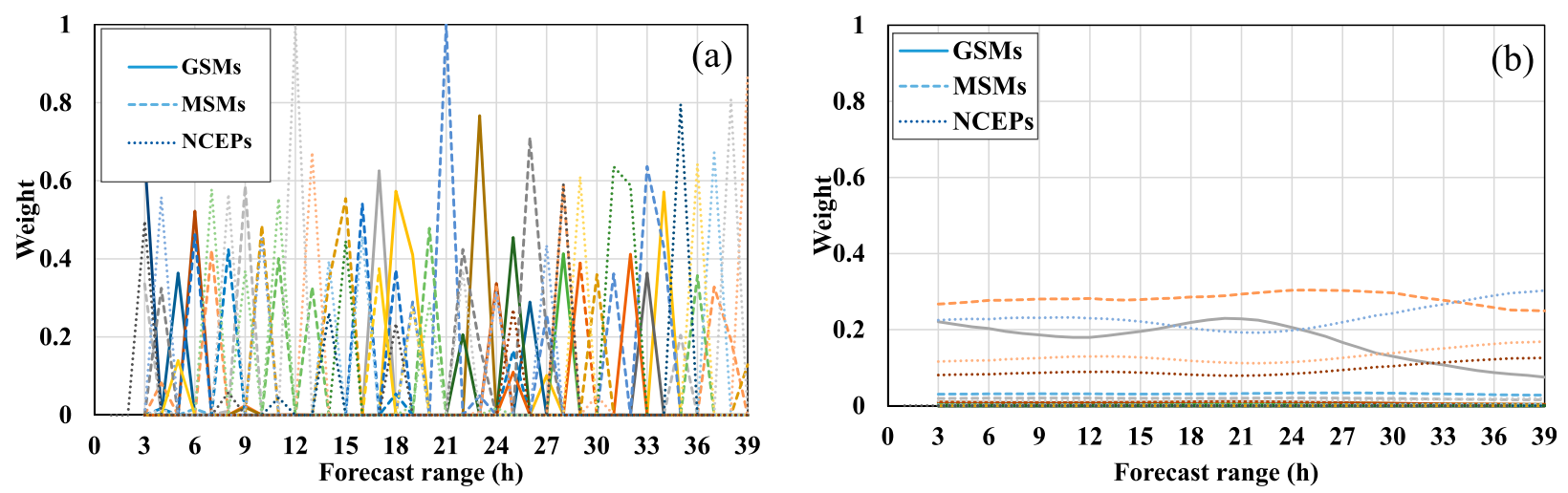

FIG. 17. Weight coefficients of ensemble members in experiment (a) N60-33 and (b) N60X3-33 for each forecast lead time from 3 to $39 \mathrm{~h}$ at Toyama station initialized at 0000 UTC 9 Sep 2018. Subensembles are distinguished by line type, and members within each subensemble are distinguished by color.

The RMSEs averaged over the forecast range and over all initial times and stations for both experiments were 1.21496 for the N60-33 and 1.18916 for the N60X3-33, indicating that the extension technique has a smaller RMSE.

These results suggest that the extension in this paper is effective for large ensembles including subensembles. It will be interesting to see if this extension is also effective for larger model ensembles.

Acknowledgments. I thank the anonymous reviewers for pointing out inadequacies in the first draft and for their helpful suggestions to strengthen the conclusions of this paper. I also thank the editor for her support.

\section{REFERENCES}

Berrocal, V. J., A. E. Raftery, and T. Gneiting, 2007: Combining spatial statistical and ensemble information in probabilistic weather forecasts. Mon. Wea. Rev., 135, 1386-1402, https:// doi.org/10.1175/MWR3341.1.

Bishop, C. M., 2010: Pattern Recognition and Machine Learning. Springer, $738 \mathrm{pp}$.

Bishop, C. H., and K. T. Shanley, 2008: Bayesian model averaging's problematic treatment of extreme weather and a paradigm shift that fixes it. Mon. Wea. Rev., 136, 4641-4652, https:// doi.org/10.1175/2008MWR2565.1.

Chmielecki, R. M., and A. E. Raftery, 2011: Probabilistic visibility forecasting using Bayesian model averaging. Mon. Wea. Rev., 139, 1626-1636, https://doi.org/10.1175/2010MWR3516.1.

Dempster, A. P., N. M. Laird, and D. B. Rubin, 1977: Maximum likelihood from incomplete data via the EM algorithm. J. Roy. Stat. Soc., 39B, 1-22, https://doi.org/10.1111/j.2517-6161.1977.tb01600.x.

Eide, S. S., J. B. Bremnes, and I. Steinsland, 2017: Bayesian model averaging for wind speed ensemble forecasts using wind speed and direction. Wea. Forecasting, 32, 2217-2227, https://doi.org/ 10.1175/WAF-D-17-0091.1.

Hersbach, H., 2000: Decomposition of the continuous ranked probability score for ensemble prediction systems. Wea. Forecasting, 15, 559-570, https://doi.org/10.1175/1520-0434(2000) $015<0559$ :DOTCRP $>2.0$. CO 2 .

Japan Meteorological Agency, 2019: Outline of the operational numerical weather prediction at the Japan Meteorological Agency. Appendix to WMO Tech. Progress Rep. on the
Global Data-processing and Forecasting System and Numerical Weather Prediction, Tokyo, Japan Meteorological Agency, accessed 12 January 2021, https://www.jma.go.jp/jma/jma-eng/ jma-center/nwp/outline2019-nwp/index.htm.

Ji, L. Y., X. F. Zhi, S. P. Zhu, and K. Fraedrich, 2019: Probabilistic precipitation forecasting over East Asia using Bayesian model averaging. Wea. Forecasting, 34, 377-392, https://doi.org/ 10.1175/WAF-D-18-0093.1.

Kleiber, W., A. E. Raftery, J. Baars, T. Gneiting, C. F. Mass, and E. Grimit, 2011: Locally calibrated probabilistic temperature forecasting using geostatistical model averaging and local Bayesian model averaging. Mon. Wea. Rev., 139, 2630-2649, https://doi.org/10.1175/2010MWR3511.1.

Kunii, M., and Coauthors, 2011: Verification and intercomparison of mesoscale ensemble prediction systems in the Beijing 2008 Olympics Research and Development Project. Tellus, 63A, 531-549, https://doi.org/10.1111/j.1600-0870.2011.00512.x.

Liu, J. G., and Z. H. Xie, 2014: BMA probabilistic quantitative precipitation forecasting over the Huaihe basin using TIGGE multimodel ensemble forecasts. Mon. Wea. Rev., 142, 15421555, https://doi.org/10.1175/MWR-D-13-00031.1.

Möller, A., A. Lenkoski, and T. L. Thorarinsdottir, 2013: Multivariate probabilistic forecasting using ensemble Bayesian model averaging and copulas. Quart. J. Roy. Meteor. Soc., 139, 982-991, https://doi.org/10.1002/qj.2009.

Mori, Y., N. Fujikane, and T. Inoue, 2021: Ongoing development of methods to combine forecast guidance outputs using artificial intelligence technology at the Japan meteorological agency. 20th Conf. on Artificial Intelligence for Environmental Science, Amer. Meteor. Soc., 12.10, https://ams.confex.com/ams/ 101ANNUAL/meetingapp.cgi/Paper/382558.

Palmer, T., 2018: The ECMWF ensemble prediction system: Looking back (more than) 25 years and projecting forward 25 years. Quart. J. Roy. Meteor. Soc., 145, 12-24, https://doi.org/ 10.1002/qj.3383.

Raftery, A. E., T. Gneiting, F. Balabdaoui, and M. Polakowski, 2005: Using Bayesian model averaging to calibrate forecast ensembles. Mon. Wea. Rev., 133, 1155-1174, https://doi.org/ 10.1175/MWR2906.1.

Schmeits, M. J., and K. J. Kok, 2010: A comparison between raw ensemble output, (modified) Bayesian model averaging, and extended logistic regression using ECMWF ensemble precipitation reforecasts. Mon. Wea. Rev., 138, 4199-4211, https:// doi.org/10.1175/2010MWR3285.1. 
Sloughter, J. M., A. E. Raftery, T. Gneiting, and C. Fraley, 2007: Probabilistic quantitative precipitation forecasting using Bayesian model averaging. Mon. Wea. Rev., 135, 3209-3220, https://oi.org/ 10.1175/MWR3441.1.

—, T. Gneiting, and A. E. Raftery, 2013: Probabilistic wind vector forecasting using ensembles and Bayesian model averaging. Mon. Wea. Rev., 141, 2107-2119, https://doi.org/ 10.1175/MWR-D-12-00002.1.

Song, X. J., Y. J. Zhu, J. Y. Peng, and H. Guan, 2018: Improving multi-model ensemble forecasts of tropical cyclone intensity using Bayesian model averaging. J. Meteor.
Res., 32, 794-803, https://doi.org/10.1007/s13351-0187117-7.

Wilks, D., 2011: Statistical Methods in the Atmospheric Sciences. 3rd ed. International Geophysics Series, Vol. 100, Academic Press, 704 pp.

_ 2014: Multivariate ensemble Model Output Statistics using empirical copulas. Quart. J. Roy. Meteor. Soc., 141, 945-952, https://doi.org/10.1002/qj.2414.

Wilson, L. J., S. Beauregard, A. E. Raftery, and R. Verret, 2007: Calibrated surface temperature forecasts from the Canadian ensemble prediction system using Bayesian model averaging. Mon. Wea. Rev., 135, 1364-1385, https://doi.org/10.1175/MWR3347.1. 